estimating burden of cocaine or hallucinogen use disorders in common Musculoskeletal diseases (MSDs) are lacking.

Objectives: To assess national time-trends in cocaine use and hallucinogen use disorders in people with MSDs

Methods: This study used the U.S. National Inpatient Sample (NIS), a de-identified national all-payer inpatient health care database (https://www.hcup-us.ahrq gov/nisoverview.jsp) from 1998-2014. The NIS is a $20 \%$ stratified sample of hospital discharges in the U.S. It is commonly used to derive national estimates of hospitalization and outcomes. Cocaine or hallucinogen use disorder hospitalization was defined in a validated approach as the presence of the following International Classification of Diseases, Ninth Revision, Clinical Modification (ICD-9-CM) diagnostic codes: cocaine use disorder, 304.2x, or 305.6x; and hallucinogen use disorder, $304.5 x$ or $305.3 x$; hospitalizations for drug use in remission, drug counseling, rehabilitation or detoxification were excluded, as in previous studies. MSDs were identified based on the respective ICD-9 codes, a validated approach (5-9), in non-primary position: Gout: 274.xx; rheumatoid arthritis (RA): 714.xx; Fibromyalgia: 729.1; osteoarthritis (OA): 715.xx; or low back pain (LBP): 724.

Results: In 1998-2000, the highest frequency of cocaine use hospitalizations was in people with LBP: LBP $(n=5,914)$, followed by OA $(n=4,931)$, gout $(n=2,093)$, RA ( $n=2,026)$, and fibromyalgia $(n=1,620)$. In 2013-2014, the order changed slightly with OA $(n=22,185)$, followed by LBP $(n=16,810)$, gout $(n=10,570)$, RA $(n=8,975)$, and fibromyalgia $(n=5,680)$. Respective rates per 1 million U.S. NIS hospitalizations in 2013-2014 and the relative increase from 1998-2000 to 20132014 were: Gout, 10.2 (increase, 4.1-fold); OA, 21.4 (3.5-fold); fibromyalgia, 5.48 (2.5-fold); RA, 8.66 (3.4-fold); and LBP, 16.22 (1.8-fold; Figure 1).

In 1998-2000, hallucinogen use disorder hospitalizations were as follows: LBP $(n=176)$, followed by OA $(n=63), R A(n=42)$, fibromyalgia $(n=41)$ and gout $(n<10$; cells with frequency of 10 of fewer are reported as $<10$ per NIS guidance). In 2013-2014, the frequency order was the similar, with the highest numbers for LBP $(n=525)$ followed by OA $(n=400)$, RA $(n=395)$, gout $(n=135)$ and fibromyalgia $(n=125)$. Respective rates per 1 million US NIS hospitalizations in 2013-2014 and the relative increase from 1998-2000 to 2013-2014 were: Gout, 0.13 (increase, 25-fold); OA, 0.39 (5.5-fold); fibromyalgia, 0.12 (2-fold); RA, 0.38 (8.5-fold); and LBP, 0.51 (2-fold; Figure 1).

Conclusion: This study confirmed an increasing rate of both, cocaine use and hallucinogen use disorder hospitalizations in people with 5 MSDs over a 17-year period from 1998-2014 in the U.S.
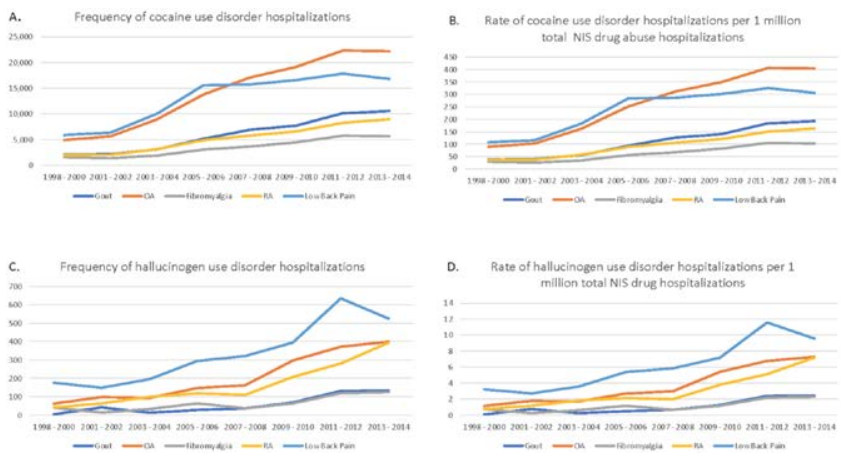

Figure 1. Time-trends in the rates of hospitalization with cocaine use and hallucinogen use disorder (A), non-home discharge (B), and in-hospital mortality (C) per 100,000 NIS hospitalization claims. The $x$-axis shows rate per 100,000 NIS hospitalization claims and the $y$-axis the study periods

Acknowledgements: I thank John D. Cleveland, MS of the University of Alabama at Birmingham for performing data analyses according to the protocol. Disclosure of Interests: Jasvinder Singh Shareholder of: JAS owns stock options in TPT Global Tech, Vaxart pharmaceuticals and Charlotte's Web Holdings, Inc. JAS previously owned stock options in Amarin, Viking and Moderna pharmaceuticals., Consultant of: JAS has received consultant fees from Crealta/Horizon, Medisys, Fidia, UBM LLC, Trio Health, Medscape, WebMD, Adept Field Solutions, Clinical Care options, Clearview healthcare partners, Putnam associates, Focus forward, Navigant consulting, Spherix, Practice Point communications, the National Institutes of Health and the American College of Rheumatology. JAS is on the speaker's bureau of Simply Speaking. JAS is a member of the executive of Outcomes Measures in Rheumatology (OMERACT), an organization that develops outcome measures in rheumatology and receives armslength funding from 12 companies.

DOI: 10.1136/annrheumdis-2021-eular.610

\section{POS0015 \\ PREVALENCE OF NEUROPATHIES IN RHEUMATIC AND MUSCULOSKELETAL DISEASES}

R. Hasseli ${ }^{1}$, M. Tschernatsch ${ }^{2,3}$, N. Heimann ${ }^{1}$, E. Neumann ${ }^{1}$, T. Gerriets ${ }^{2,4}$, J. Allendörfer ${ }^{5}$, T. Ruck ${ }^{6}$, A. Schänzer ${ }^{7}$, U. Müller-Ladner ${ }^{1} .{ }^{1}$ Justus-LiebigUniversity Giessen, Campus Kerckhoff, Department of Rheumatology and
Clinical Immunology, Bad Nauheim, Germany; ${ }^{2}$ Justus-Liebig-University Giessen, Department of Neurology, Giessen, Germany; ${ }^{3}$ Gesundheitszentrum Wetterau, Department of Neurology, Bad Nauheim, Germany;

${ }^{4}$ Gesundheitszentrum Wetterau, Department of Neurology, Friedberg, Germany; ${ }^{5}$ Asklepios Hospital, Department of Neurology, Bad Salzhausen, Germany; ${ }^{6}$ Heinrich-Heine-University Duesseldorf, Department of Neurology, Giessen, Germany; ${ }^{7}$ Justus-Liebig-University Giessen, Institute of Neuropathology, Giessen, Germany

Background: In rheumatic and musculoskeletal diseases (RMDs), peripheral neurons can be affected, which can result in sensory symptoms like pain, burning, tingling, numbness and motor symptoms like muscle-atrophy or even paresis. More detailed knowledge about the prevalence and the cause of neuropathy (NP) in RMD are urgently needed, especially as RMD patients may develop different subtypes of NP.

Objectives: The aim of this project was to assess the prevalence and the individual types of NP in rheumatoid arthritis (RA), spondyloarthritis (SpA) and systemic sclerosis (SSc) patients, and to elucidate the clinical, neurophysiological and neuropathologic features of associated NP.

Methods: Baseline questionnaires and neurological and physical examination were used to elucidate the presence of neuropathic pain and autonomic dysfunction. Laboratory tests were performed to exclude other causes for NP. Electrophysiological tests were performed to differentiate demyelinating from axonal large fiber (LF)NPs. Additionally, skin biopsies were used to detect an involvement of small fibres (SF).

Results: A total of 31 patients (median age 64 years (range 43-75)) were included. The majority of patients were female $(90 \%)$. The mean disease dura tion was 10 years (1-41 years). More than $50 \%$ of the patients were diagnosed with RA, 7 with SpA and 6 with SSc. Of 31 patients, 48\% (15/31) had clinical signs of NP and of those, neurophysiological examination showed 14 axonal 2 , demyelinating and 4 mixed types. A combined LFNP and SFNP was present in $35 \%(11 / 31)$ of the patients. In 4 patients, only a SFNP was detectable, and in only two patients, no NP was detectable.

Conclusion: NP was detectable in $94 \%$ (29/31) of the RMD patients, with LFNP predominating. This high proportion of NP in RMD suggests a surprisingly high coincidence of both diseases.

Table 1. Subtypes of NP in RMD

\begin{tabular}{lc}
\hline & Number of patients \\
\hline Axonal NP & $14 / 31(45 \%)$ \\
Demyelinating NP & $2 / 31(6 \%)$ \\
Mixed axonal and demyelinating NP & $4 / 31(12 \%)$ \\
Sensory NP & $9 / 31(26 \%)$ \\
Sensorimotor NP & $5 / 31(10 \%)$ \\
Motor NP & $1 / 31(3 \%)$ \\
\hline
\end{tabular}

Disclosure of Interests: None declared.

DOI: 10.1136/annrheumdis-2021-eular.3931

\section{POS0016 OF PATIENTS WITH OSTEOPOROTIC VERTEBRAL FRACTURES AS A BASIS FOR REHABILITATION PROGRAMS DEVELOPING}

L. Marchenkova ${ }^{1}$, V. Vasileva ${ }^{1}$, M. Eryomushkin ${ }^{1} .{ }^{1}$ National Medical Research Center for Rehabilitation and Balneology of Ministry of Health of Russian Federation, Rehabilitation Department for Somatic Patients, Moscow, Russian Federation

Background: Due to the demand for special rehabilitation programs for patients with osteoporotic vertebral fractures (VFs), it is of interest to study the functional abilities of those patients. The scientific hypothesis suggests that osteoporotic VFs would cause muscle weakness, muscle dysfunction and conditional disturbances.

Objectives: to estimate muscle strength, motor function and coordination disorders in patients with VFs in the setting of systemic osteoporosis as a basis for rehabilitation programs developing.

Methods: 120 patients aged 43-80 with primary osteoporosis were enrolled. Study group comprised of 60 subjects (56 women, 4 men) with at least 1 VF confirmed by X-rays. Control group included 60 subjects (56 women, 4 men) with osteoporosis but without any osteoporotic fracture. The examination program included back muscles tenzodynamometry, balance tests and stabilometry. Results: Muscle strength deficiency was estimated in study group in trunk flexors (TF) $-40.9 \%$ and in trunk extensors $(\mathrm{TE})-18.1 \%$ with an adequate function of the left lateral flexors (LLF) and in right lateral flexors (RLF). Patients with VFs had the lower muscle strength vs controls of TE $(15.64 \pm 9.8 \mathrm{vs} 27.73 \pm 9.9 \mathrm{~kg}$ $\mathrm{p}=0.00002)$, TF $(14.61 \pm 8.98$ vs $21.28 \pm 8.38 \mathrm{~kg}, \mathrm{p}=0.0006), \operatorname{LLF}(13.10 \pm 7.2$ vs 
$24.06 \pm 8.9 \mathrm{~kg}, \mathrm{p}=0.005)$ and RLF $13.44 \pm 7.43$ vs $24.26 \pm 7.65 \mathrm{~kg}, \mathrm{p}=0.0003$ ). Patients with VFs lose their balance faster during one-leg-standing test with open eyes $(5.0[1.0 ; 10.0]$ vs $7.5[5.0 ; 10.5] \mathrm{sec}$ in control group, $p=0.03)$ and with closed eyes $(2.0[0 ; 3.0]$ vs $3.5[3.0 ; 5.0] \mathrm{sec}, \mathrm{p}=0.04)$. Fukuda-Unterberger test showed greater side dislocation in study group $-40^{\circ}[25 ; 45]$ vs controls $30^{\circ}[10$; $45],(p=0.02)$. According to stabilometry study group was characterized vs control group by lower balance coefficient with open eyes $(77.2 \pm 7.6$ vs $85.7 \pm 9.4 \%$, $\mathrm{p}=0.002)$ and with closed eyes $(67.1 \pm 9.8$ vs $73.4 \pm 9.9 \%, p=0.03)$, greater sagittal displacement $(6.8[2.1 ; 37.7]$ vs $4.8[1.8 ; 10.7] \mathrm{mm}, \mathrm{p}=0.025)$ and deviation in the saggital plane $(1.2[-1.07 ; 1.5]$ vs $-1.2[-1.5 ; 1.2] \mathrm{mm}, \mathrm{p}=0.01)$, and also less pressure center velocity $(9.51 \pm 4.4$ vs $7.1 \pm 2.7 \mathrm{~mm} / \mathrm{sec}, \mathrm{p}=0.009)$.

Conclusion: Osteoporotic VFs are associated with reduction of trunk muscles strength and negatively affect static and dynamic balance function that should be taken into account when developing rehabilitation programs for these patients.

Disclosure of Interests: None declared.

DOI: 10.1136/annrheumdis-2021-eular.4260

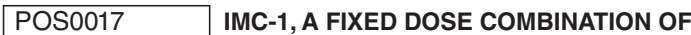 FAMCICLOVIR AND CELECOXIB, IMPROVES COMMON SYMPTOMS ASSOCIATED WITH FIBROMYALGIA IN ADDITION TO PAIN: POST HOC ANALYSIS OF A PHASE 2A TRIAL}

W. Pridgen ${ }^{1}$, C. Duffy ${ }^{2}$, J. F. Gendreau ${ }^{3}$, R. M. Gendreau ${ }^{4}{ }^{1}$ Tuscaloosa Surgical Associates, P.C., LLC, Tuscaloosa, United States of America; ${ }^{2}$ University of Alabama, Department of Biological Sciences, Tuscaloosa, United States of America; ${ }^{3}$ Gendreau Consulting, LLC, Poway, United States of America; ${ }^{4}$ Virios Therapeutics, Alpharetta, United States of America

Background: Fibromyalgia is a chronic disease characterized by widespread pain and severe fatigue that may be triggered by reactivation of latent herpes simplex virus type 1 (HSV-1). In a Phase 2a proof of concept trial, IMC-1 (a fixed dose combination of famciclovir and celecoxib) demonstrated greater tolerability and statistically significant reduction in pain compared with placebo, as measured by change from baseline to week 16 in 24-hour recall pain intensity on an 11-point Numerical Rating Scale (NRS) and 7-day recall pain intensity on the 11-point pain item on the Revised Fibromyalgia Impact Questionnaire (FIQ-R). Objectives: In this post hoc analysis, we evaluated the effects of IMC-1 compared with placebo on other fibromyalgia symptoms, including lack of energy, stiffness, problems with sleep, problems with memory, depression, and anxiety. Methods: In the double-blind, multi-center, placebo-controlled trial, male or female patients 18-70 years of age who met diagnostic criteria for fibromyalgia and had at baseline a 24-hour recall average pain intensity score between 4 and 9 on the NRS were randomized 1:1 to 16 weeks of treatment with IMC-1 or placebo. Mean changes from baseline to week 16 in FIQ-R symptom scores were analyzed using a Mixed-Effect Model Repeated Measure (MMRM) model with treatment as the main effect, and investigative site and baseline FIQ-R symptom scores as covariates. Results: A total of 143 patients were enrolled and randomized to treatment with IMC-1 $(n=69)$ or placebo $(n=74)$. Baseline demographic and clinical characteristics were comparable between treatment groups; the majority of patients were Caucasian $(95.8 \%)$ and female $(93.7 \%)$ with a mean age of $\sim 49$ years. Compared with placebo, treatment with IMC-1 resulted in statistically significant improvements in the FIQ-R symptom scores of stiffness (least squares mean change from baseline -0.96 vs. $-1.92, P=0.03)$, sleep quality $(-0.76$ vs. $-1.76, P=0.039)$, depression $(-0.44$ vs. $-1.33, P=0.016)$, and anxiety $(-0.30$ vs. $-1.69, P<0.001)$, but not in energy level (-0.67 vs. $-1.29, P=0.115)$ or memory problems $(-0.71$ vs. $-1.24, P=0.165)$.

Conclusion: In addition to alleviation of chronic pain, treatment with IMC-1 appears to be effective in improving many of the other symptoms often associated with fibromyalgia. Further clinical trials are warranted.

REFERENCES:

[1] Pridgen WL, Duffy C, Gendreau JF, Gendreau RM. A famciclovir + celecoxib combination treatment is safe and efficacious in the treatment of fibromyalgia. J Pain Res. 2017;10:451-460.

Disclosure of Interests: William Pridgen Consultant of: Virios Therapeutics, Carol Duffy Consultant of: Virios Therapeutics, Grant/research support from: The University of Alabama, Department of Biological Sciences has received financial research support from Innovative Med Concepts (now Virios Therapeutics) in the form of two Sponsored Research Agreements., Judy F. Gendreau Consultant of: Tonix, Dare Bioscience, Virios Therapeutics, R. Michael Gendreau Consultant of: Tonix, Teva, Swing Therapeutics, Dare Bioscience, Employee of: Virios Therapeutics.

DOI: 10.1136/annrheumdis-2021-eular.1424

\section{POS0018 INVESTIGATING VIRTUAL IMMERSIVE EXPERIENCES IN THE MANAGEMENT OF CHRONIC PAIN - THE VIPA STUDY (PRELIMINARY RESULTS)}

J. Tsigarides ${ }^{1}$, V. Grove ${ }^{1}$, D. Sethi ${ }^{1}$, J. Chipping ${ }^{1}$, S. Miles ${ }^{1}$, N. Shenker ${ }^{2}$,

S. Sami ${ }^{1}$, A. Macgregor ${ }^{1} .{ }^{1}$ University of East Anglia, Norwich Medical School,

Norwich, United Kingdom; ${ }^{2}$ Addenbrookes University Hospital, Rheumatology,

Cambridge, United Kingdom

Background: Chronic pain is debilitating and prevalent. Current non-pharmacological management of pain conditions such as Fibromyalgia Syndrome (FMS) are labour intensive to implement and poorly available, especially during the pandemic. There is an urgent need to develop widely adoptable, innovative treatment options for pain cohorts.

Virtual reality (VR) provides an innovative therapeutic tool, immersing users within a three-dimensional, interactive virtual environment with use of a headmounted display (HMD). Beneficial effects of VR have been demonstrated in acute pain ${ }^{1}$, with limited studies in chronic pain. Given the variation of available VR technologies, it is vital to investigate the impact of different VR characteristics on acceptability in specific chronic pain cohorts.

Objectives: This feasibility study aims to establish the acceptability of four different VR technologies in patients with FMS whilst undertaking a single interactive VR experience.

Methods: Patients with FMS were recruited through outpatient clinics at the Norfolk and Norwich University Hospital. Baseline questionnaires were used including the McGill pain questionnaire (MPQ-SF), pain visual analogue scale (VAS) and Revised Fibromyalgia Impact Questionnaire (FIQR). Subjective experience questionnaires collected acceptability data with 7-point Likert scale rating questions (strongly disagree to strongly agree). The simulation sickness questionnaire (SSQ) gained side-effect data (total severity score: $0-235$ ). Categorical data were described using frequencies; and continuous data using mean and standard deviation. Likert-scale data were dichotomised (rating $\leq 3$ : disagree, rating $\geq 5$ : agree).

Four VR systems representing the spectrum of commercially available technologies were used (seen in Figure 1). These possess different characteristics including screen resolution, processor speed, weight, strap and controller type. The VR experience used with each headset was co-developed alongside industry partners (Orbital Global). Participants are immersed within a naturalistic environment, situated on a wooden boat travelling slowly along a calm river surrounded by trees and hills. The interactive element involves the user shooting targets that appear using handheld controllers.

Results: 13 patients with FMS were included (mean age 41.8ะ15.6, 92.3\% female). Most had severe disease (mean FIQR 67.8 14.1 ) with moderate self-reported pain at baseline (mean MPQ $25.5 \pm 8.8$, VAS $6.0 \pm 1.7$ ). Most had no previous VR exposure (69.2\%). $100 \%$ of participants agreed that they would be open to using VR for future pain management (mean rating $6.5 \pm 0.7$ ) and that they would use VR regularly at home (mean rating 6.5 \pm 0.7 ). VR HMD comfort and enjoyment data are presented in Table 1. Mean ratings of comfort were high across the four HMDs (Gear VR: $4.9 \pm 1.7$, Oculus Go: $4.5 \pm 1.8$, Oculus Quest $5.3 \pm 1.9$, Oculus Rift $6.6 \pm 0.5$ ). Mean ratings of enjoyment with each HMD were also high (Gear VR: $5.4 \pm 1.6$, Oculus Go: $5.4 \pm 1.8$, Oculus Quest: $5.6 \pm 1.9$, Oculus Rift S: $6.6 \pm 0.5$ ). Low levels of side effects were described with mean SSQ total scores ranging from 20.1 \pm 16.8 (Oculus Rift S) to $38.0 \pm 23.9$ (Gear VR).

Conclusion: Preliminary results indicate that FMS patients find VR acceptable, describing high ratings of comfort and enjoyment across the VR HMD spectrum. Side-effect frequency was low, with most settling after HMD removal. All participants were open to future use of VR for home-based pain management. REFERENCES:

[1] Dascal J, Reid M, Ishak WW, Spiegel B, Recacho J, Rosen B, Danovitch I. Virtual reality and medical inpatients: A systematic review of randomized, controlled trials. Innov Clin Neurosci 2017;14(1-2):14-21

Table 1. Subjective experience results across VR HMDs

\begin{tabular}{lcc}
\hline & Mean Likert scale ratings (\% agreement: rating $\geq 5)$ \\
\hline VR HMD & $\begin{array}{c}\text { Overall, I found the VR experience using this Overall, I enjoyed using this } \\
\text { VR headset }\end{array}$ \\
\hline Gear VR & $4.9 \pm 1.7(62 \%)$ & $5.4 \pm 1.6(77 \%)$ \\
Oculus Go & $4.5 \pm 1.8(54 \%)$ & $5.4 \pm 1.8(75 \%)$ \\
Oculus Quest & $5.3 \pm 1.9(77 \%)$ & $5.6 \pm 1.9(100 \%)$ \\
Oculus Rift S & $6.6 \pm 0.5(100 \%)$ & $6.6 \pm 0.5(100 \%)$ \\
\hline
\end{tabular}

